\title{
SOME REVERSE $l_{p}$-TYPE INEQUALITIES INVOLVING CERTAIN QUASI MONOTONE SEQUENCES
}

\author{
Mikhail K. Potapov, Faton M. Berisha, \\ Nimete SH. BERISHA AND RESHAD KADRIU
}

Abstract. In this paper, we give some $l_{p}$-type inequalities about sequences satisfying certain quasi monotone type properties. As special cases, reverse $l_{p}$-type inequalities for non-negative decreasing sequences are obtained. The inequalities are closely related to Copson's and Leindler's inequalities, but the sign of the inequalities is reversed.

Mathematics subject classification (2010): Primary 47A30; Secondary 26D15.

Keywords and phrases: $l_{p}$-type, Copson, Leindler, inequalities, quasi, lacunary, geometrically, monotone sequences.

\section{REFERENCES}

[1] E. T. Copson, Note on Series of Positive Terms, J. London Math. Soc., S1-3 (1):49, 1928.

[2] P. GAO, On $l^{p}$ norms of factorable matrices, ArXiv e-prints, January 2013, 1301.3262.

[3] G. H. Hardy, J. E. Littlewood, And G. Pólya, Inequalities, Cambridge Mathematical Library. Cambridge University Press, Cambridge, 1988. Reprint of the 1952 edition.

[4] A. A. Konyushrov, Best approximations by trigonometric polynomials and fourier coefficients, Mat. Sb. N.S., 44 (3): 53-84, 1958, (Russian).

[5] L. LEINDLER, Über verschiedene Konvergenzarten trigonometrischer Reihen, III. Bedingungen in der Metrik von $L^{p}$, Acta Sci. Math. (Szeged), 27: 205-215, 1966.

[6] L. LeINDLER, Generalization of inequalities of Hardy and Littlewood, Acta Sci. Math. (Szeged), 31: 279-285, 1970.

[7] L. LEINDLER, Power-monotone sequences and Fourier series with positive coefficients, JIPAM. J. Inequal. Pure Appl. Math., 1 (1): Article 1, 10, 2000.

[8] M. K. Potapov, A certain imbedding theorem, Mathematica (Cluj), 14 (37): 123-146, 1972.

[9] M. K. POTAPOV AND M. Q. BERISHA, Moduli of smoothness and the Fourier coefficients of periodic functions of one variable, Publ. Inst. Math. (Beograd) (N.S.), 26 (40): 215-228, 1979.

[10] M. K. Potapov, B. V. Simonov, And S. Yu. Tikhonov, Mixed moduli of smoothness in $L_{p}$, $1<p<\infty$ : a survey, Surv. Approx. Theory, 8: 1-57, 2013.

[11] T. M. Vukolova, Some properties of trigonometric series with monotonic coefficients, Vestnik Moskov. Univ. Ser. I Mat. Mekh., (6): 18-23, 110, 1984. 